\title{
ANCHIS (CALATAYUD, ZARAGOZA): UNA CANTERA DE BILBILIS
}

\author{
ISIDRO AGUILERA ARAGON \\ Arqueo-Expert, S. L., Zaragoza \\ Miguel CISNEROS CUNCHILLOS \\ Dpto. de Ciencias Históricas (Arqueología). \\ Universidad de Cantabria. \\ JOSEP GISBERT AGUILAR \\ Dpto de Geología. Universidad de Zaragoza
}

\section{Resumen}

En el estudio de impacto ambiental llevado a cabo en diciembre de 1994 por Arqueo-Expert, S.L., con ocasión del proyecto de construcción de la línea del AVE Madrid-Zaragoza, se descubrió una cantern con restos de extracción en el paraje de Anchís (Calatayud, Zaragoza). En ella, se observan diversos bloques en diferente fase del proceso de extracción, así como improntas de algunos ya extraidos. Dado que la distancia, en línea recta, entre la cantera y el Munictpium Augusta Bilbilis es de un kilometro, todo indica que esta caliza micrítica fue explotada para abastecer a los edificios del municipio romano, lo que, además, está comprobado al identificarse esta roca en columnas y capiteles del teatro y en las losas de la plaza del Foro. Esta cantera, la primera localizada en las proximidades del yacimiento romano, será destruida por la construcción del AVE, al coincidir con el trazado de la vía.

Summary

The environmental impact assessment of the "High Speed Train" (Madrid-Zaragoza) was carried out in December 1994 by Arqueo-Expert S.L.In this archaeological survey an ancient quarry was located at Anchis (near Calatayud).In this quarry there are several stone blocks in diferent phases of extraction and casts of 
some blocks that were extracted. This quarry is one $\mathrm{km}$ away from Municipium Augusta Bilblis, and it is possible that its micritic limestone was quarried to supply the roman town of Bibilis.In fact, we have identified this kind of rock in columns and capitals from the theater, and in the flagstones of the forum. This quany (the first one discovered near the roman town) will be destroyed to built the new railway.

\section{INTRODUCCIÓN}

La investigación sobre la procedencia del material pétreo usado en el Municipium Augusta Bilbilis (Calatayud, Zaragoza) se ha venido desarrollando desde hace más de una década en dos direcciones: una, la identificación de los marmora o rocas ornamentales empleadas en la arquitectura, escultura y epigrafía, y otra, la búsqueda de las fuentes de extracción del material local (MARTiN-BUENO y CISNEROS CUNCHILIOS, 1985, 875-879; CISNEROS CUNCHILLOS, 1989, 61-63, y CISNEROS CUNCHILLOS y MARTiN-BUENO, 1994, 197-198). Estas últimas plantean una mayor dificultad de ubicación, dada la carencia de datos que se poseen sobre las mismas. En este sentido se ha trabajado con la hipótesis de que son materiales utilitarios, cuyas canteras dejaron de explotarse al concluir las obras, al agotarse o siguieron siendo beneficiadas en épocas posteriores, sin dejar huellas localizables. Por todo ello, las identificaciones siempre han hecho referencia a áreas-fuente cercanas al yacimiento (CISNEROS CuNCHILlos, 1989, 63). Además, los trabajos de prospección, encaminados en esta vía, en las zonas próximas a la excavación nunca dieron resultados positivos; por consiguiente, sốlo el azar dejaba abierta la posibilidad de hallazgo de esas canteras, siendo escasas las posibilidades de tal existencia, dados los antecedentes citados.

Sin embargo, en el mes de diciembre de 1994, con motivo del proyecto de construcción de la línea del Tren a Alta Velocidad (AVE) Madrid-Zaragoza, y al realizarse el correspondiente estudio de impacto ambiental en los tramos Calatayud-Ricla-Salillas de Jalón, que contemplaba -tal como establece el Real decreto 1131/88, art. 8- los aspectos relacionados con el patrimonio arqueológico, se descubrió y se efectuó, por parte de la empresa Arqueo-Expert, S. L., una primera documentación de la cantera que estudiamos'.

\section{LOCAIIZACIÓN}

La cantera se localiza en el término municipal de Calatayud, a cuatro kilómetros y medio al Noreste del casco urbano, en la margen derecha del río Jalón, entre éste y la antigua Carretera Nacional II, no lejos del caserío de Marivella, aunque en el paraje de Anchís; de ahí la denominación que hemos adoptado. Su posición topográfica exacta viene determinada por las siguientes coordenadas UTM: 45814.5 y 6175 , con una altitud sobre el nivel del mar de 590 mts., según la Hoja 509 - Calatayud- del Instituto Geo-

${ }^{1}$ Agradecemos a los directores de la actuación, J.Delgado y J.A.Pérez Casas de Arqueo-Expert, S.L., la amabilidad que han tenido al proporcionamos los datos sobre el yacimiento de Anclís y al Dr. Manuel MartinBueno, director de las excavaciones de Bilbilis, su inestimable colaboración. 
gráfico del Ejército. El yacimiento coincide con el punto kilométrico 4716 del proyecto del AVE.Desde un punto de vista geológico se encuentra ubicada en el borde noreste de la fosa terciaria de Calatayurd.

Los materiales terciarios aflorantes son de muro a techo un estrato calizo de $1 \mathrm{~m}$. de espesor y que lateralmente (hacia el norte) pasan a una alternancia de calizas y margas en paquetes de $0,4 \mathrm{~m}$. Sobre él existen $1,5 \mathrm{~m}$. de margas y calizas margosas blancas.No se pueden apreciar más series terciarias al estar cubiertas a techo por terrazas, coluviones y glacis cuaternarios y en el muro por coluviones de ladera.

En el estrato calizo, con porosidad macroscópica, alterna una porosidad fenestral con orra de poros ramificados con geometría de raíz y otros poros rectilíneos perpendiculares a la estratificación atribuibles también a bioturbación vegetal. Estos últimos son más frecuentes hacia el Oeste siendo más abundantes a medida que se adelgaza el estrato calcáreo. Las calizas son, de visu, micríticas y masivas, con locales brechificaciones y niveles de intraclastos subredondeados. La roca no presenta diaclasas y solo posee un aspecto friable en los puntos en los que es algo margosa y a consecuencia de la alteración meteórica.

De visu se puede clasificar como una caliza lacustre (micrita, localmente con intraclastos y/o brechoide y/o arenosa) presentando una facies muy común en las calizas del "páramo" de los terciarios continentales.

El frente de cantera se sitúa sobre el estrato basal de caliza masiva, que la acción erosiva de los barrancos que desaguan al Jalón dejó al descubierto hacia la mitad de la ladera, al excavar el relleno sedimentario terciario (figura 1). De esta forma, quienes aprovecharon la roca, como material de construcción, tuvieron fácil acceso a ella desde el flanco sur del barranco.

En el estrato de la cantera hemos identificado las siguientes microfacies ${ }^{2}$ :

Facies 1(C-1 y C-4).-Calizas micríticas finas homogéneas y algo margosas de tonos gris-amarillentos con algún intraclasto disperso, así como huellas de raíces muy ocasionales y dispersas dando macroporosidad fina (huecos de 0,1-0,5 mm.). También presenta pequeñas dendritas de pirolusita y avidez por el agua (pequeñas proporciones de sepiolita). Existen bandas estratiformes más calcáreas (de 1-2 cm de grosor y de espaciado) de color más gris y con límites difusos a la caliza algo margosa. Estos tramos más calcáreos poseen mayor macroporosidad que aún así sigue siendo pequeña.

Mineralogia: calcita y arcilla (sepiolita...)

Facies 2(C-2 y C-7). - Caliza micritica brechoide con alta macroporosidad desarrollada preferentemente sobre la matriz de las zonas brechificadas. Éstas están en relación a grietas de retracción y perforaciones de raíces. Hay también un moteado de "pseudoglei" de coloración muy tenue. La matriz de estas zonas brechificadas está rellena por intraclas-

\footnotetext{
${ }^{2}$ Hemos estudiado las muestras de roca usando láminas delgadas, réplicas en acetato, tinciones de alicerina (para calcita/dolomita), piezas pulidas para observaciones con lupa además de las observaciones sobre el terteno macro y mesoscópicas.
} 
tos $\left(\mathrm{F}_{\mathrm{m}}=0,3 \mathrm{~mm} \mathrm{~F}_{\mathrm{m}}=\right.$ tamaño medio), micrita y terrígenos $\left(\mathrm{F}_{\mathrm{m}}=0,15 \mathrm{~mm}\right)$. Puede haber amplias zonas $\sin$ brechificar de micrita con algún intraclasto disperso. Suele haber películas arcillosas de color verde alrededor de las zonas brechificadas y de los intraclastos.

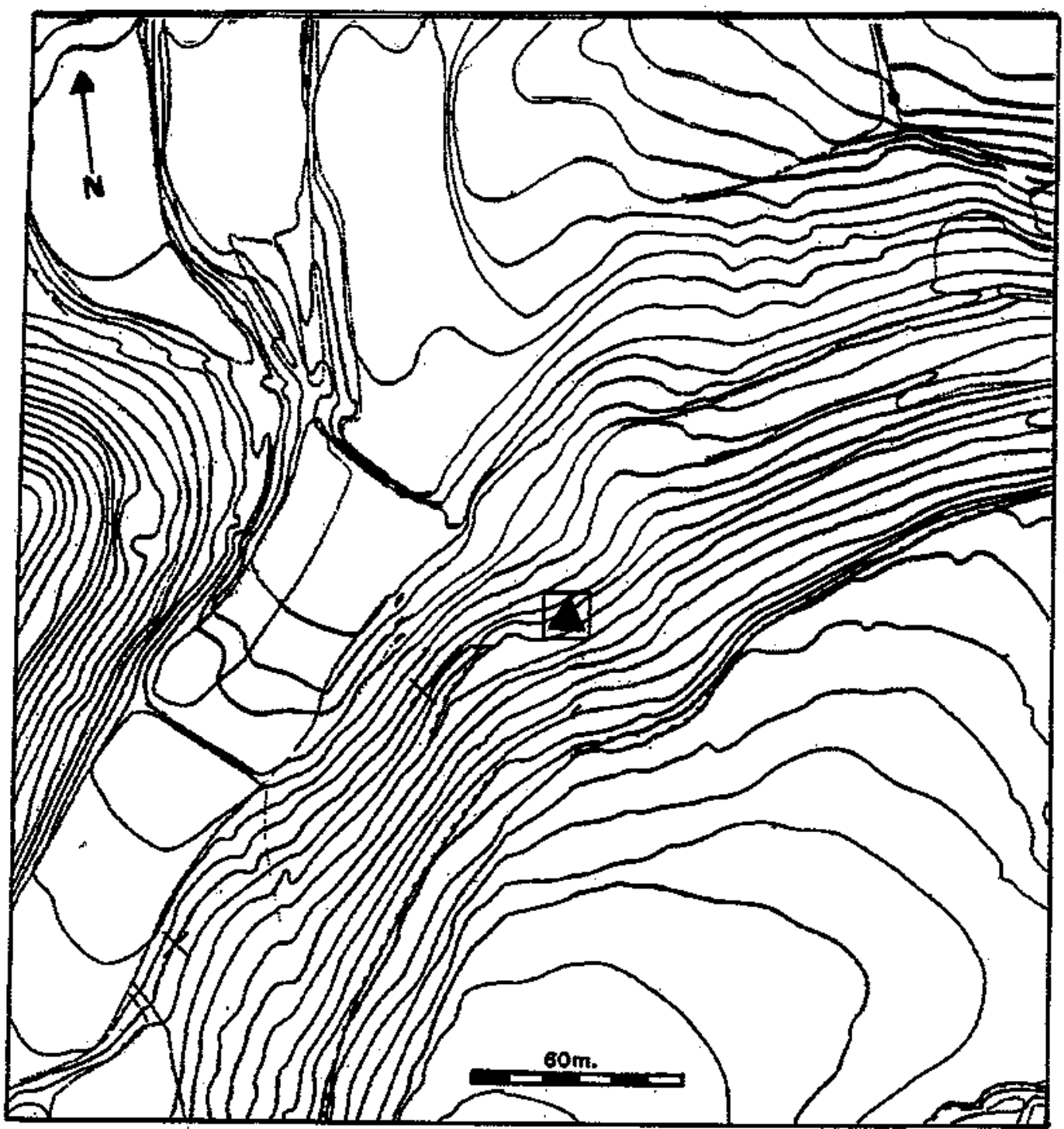

Figura 1. Ubicación de la cantera en su entorno inmediato.Equidistancia de la curvas de niveli $1 \mathrm{~m}$

Mineralogía: Calcita. En los intraclastos cuarzo, micas y fragnentos de rocas.

Facies 3(C-3).-Tránsito entre las facies $1 \mathrm{c}$ y $2 \mathrm{c}$ caracterizada por ser una micrita homogénea, sin arcilla ni terrígenos, de grano finisimo y muy duras (probablemente hay 
calcedonia dispersa) con un bandeado de color semejante al de la facies 1c y con una ligera brechificación. La fractura es concoide y suelen presentar un anillo de alteración característico 2-3 $\mathrm{mm}$ por debajo de la superficie expuesta a la meteorización.

Mineralogía: Calcita y calcedonia

Facies 4 ( $C-5$ y C-6). - Calizas arenosas con dos variedades, una con terrígenos de grano fino ( $F_{\mathrm{a}}=0,1 \mathrm{~mm}$ y $15-20 \%$ en total de terrígenos) y otra de grano medio/grueso $\left(\mathrm{F}_{\mathrm{w}}=0,5 \mathrm{~mm}\right.$. y un total de $35.40 \%$ de terrigenos). El resto de la roca es micrita parcialmente recristalizada a microesparita e intraclasto con tamaños ligeramente superiores a los terrigenos asociados.

Mineralogía:Calcita. En los intraclastos cuarzo micas y fragmentos de rocas

Facies 5 (C-8).-Micrita con intraclastos, semejante a la facies 2 pero sin brechificación. La individualización de dominios esta condicionada por una intensa bioturbación de raices verticales a través de cuyos tubos verticales existe un relleno margoso.

Mineralogía: Calcita y arcilla (probablemente sepiolita).

\section{DESCRIPCIÓN}

En la acrualidad, se observa un frente de casi $8 \mathrm{mts}$. de longitud, si bien una limpieza cuidadosa podría ampliar esta medida, en especial hacia el lado Oeste, ya que la roca es más compacta y es allí donde es mayor la cantidad de derrubios que la ocultan. Se aprecian con bastante claridad los negativos de los sillares extraídos, así como alguno que otro en fase de extracción.Tanto las dimensiones de los bloques como su proceso de elaboración, es dispar. No han aparecido materiales arqueológicos muebles en los alrededores de la cantera. Asímismo, hay que señalar que desde el propio yacimiento se pueden avistar las ruinas del municipio romano de Bilbilis, que dista un kilómetro en línea recta (figura 2).

Sólo un bloque se halla aislado de la masa rocosa y a falta del empleo de cuñas para la separación de aquélla; las dimensiones que presenta son de entre 82 y $84 \mathrm{~cm}$ de longitud -depende del lado-, de entre 41 y 46 de anchura y 54 de altura. La ranura realizada para su escisión oscila entre los $10 \mathrm{y}$ los $18 \mathrm{~cm}$ (figuras $3 \mathrm{y} 0$ ).

El resto nos permite ver con claridad las diferentes fases de la extracción de bloques regulares en una cantera. Así, podemos observar al Norte del ya citado, otro con $61 \mathrm{~cm}$ de longitud y 60 de anchura y al Este del primero, uno de $100 \mathrm{~cm}$ de longitud y de entre 50 y 52 de anchura, individualizado en dos de sus lados por las típicas ranuras, que presentan entre 10 y $18 \mathrm{~cm}$ y entre 14 y $15 \mathrm{~cm}$, a su izquierda y derecha, respectivamente. En la parte más oriental de este sector, se aprecian dos bloques todavía unidos, pero que se hubieran separado en caso de que los canteros hubieran proseguido sus trabajos, como indica el retalle de $13 \mathrm{~cm}$, uno de $90 \mathrm{~cm}$ de longitud y 78 de anchura y otro, el más exterior, de 57 de longitud y 65 de anchura. Por último, citar que en la parte más occidental, se distingue entre el manto vegetal un bloque de $105 \mathrm{~cm}$ de longitud, separado por una ranura de $17 \mathrm{~cm}$ (figuras 4 a 7 ). 


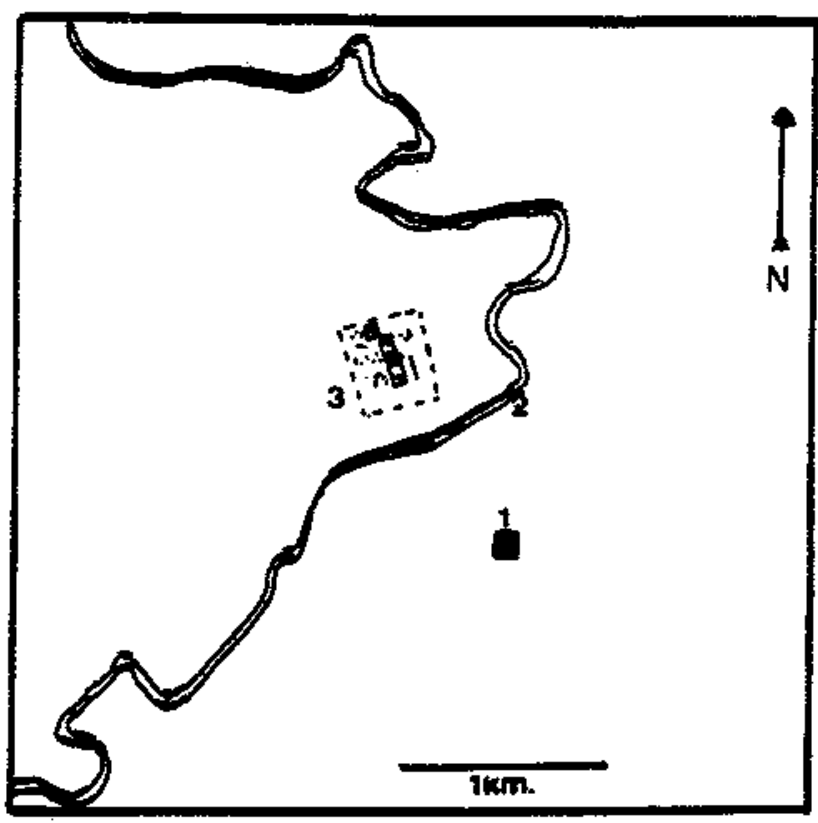

Figura 2. Situación de la cantera (1), el río jalón (2) $\mathrm{y}$ de Bibilis (3)

Como ya ha quedado expresado, en este sector se observa con claridad el proceso seguido para la extracción de bloques, que no difiere del ya conocido en otras canteras a cielo abierto, donde se trabajaba mediante el sistema de gradas. Al menos dos de los lados del bloque estaban ya libres por el arranque de otro u otros precedentes. Los contornos aproximados del bloque a arrancar son señalados por una ranura que define la línea de ruptura del lado de la pared y del lecho inferior, cuya anchura tenía aproximadamente $15 \mathrm{~cm}$ y su profundidad era variable, dependiendo de la altura del bloque a extraer. Esta operación se hacia con un pico o con cincel y martillo. En el canal y a intervalos variables se realizaban unas cavidades cuadrangulares o circulares, en las que eran colocadas las cuñas para la operación de arranque. Si éstas eran metálicas se introducian a golpe de martillo, si eran de madera se mojaban en abundancia, de forma que su dilatación hiciese ceder el bloque por la línea de ruptura ${ }^{3}$.

El cálculo de anchura mínima se ha establecido en $15 \mathrm{~cm}$ y el de profundidad en 25 , ya que es el espacio menor que necesitaba el trabajo con cincel y martillo (Kozel, 1988a, 34). En nuestro caso, como ya se ha comentado, esas ranuras oscilan entre $10 \mathrm{y}$ $18 \mathrm{~cm}$ y su profundidad parece variable, según se intuye de la disposición del manto vegetal. Aunque no se observen los orificios que se practicaban para la colocación de

${ }^{3}$ Aunque in bibliografia sobre el tema es muy amplia, puede consultarse: Mannoni $\left(1984^{2}, 69-75\right)$, Bedon (1984, 101-109), Kozelj (1988a, 31-39 y 1988b, 6) y Rockwell (1989, 169-178). 


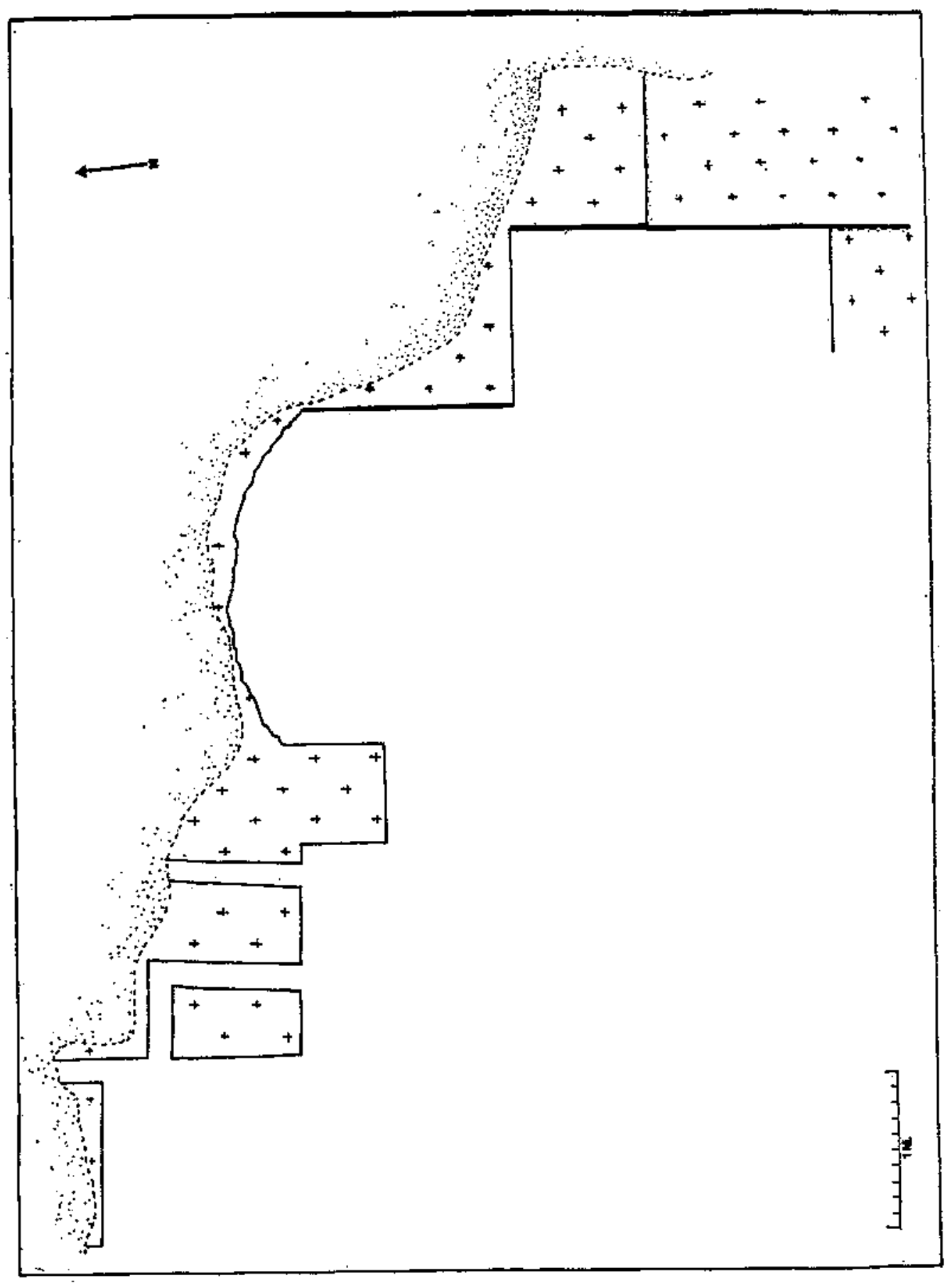

Figura 3. Planta de la cantera 
cuñas, éstas del)ieron de utilizarse en los trabajos de extracción, como era habitual y se hat explicatos.

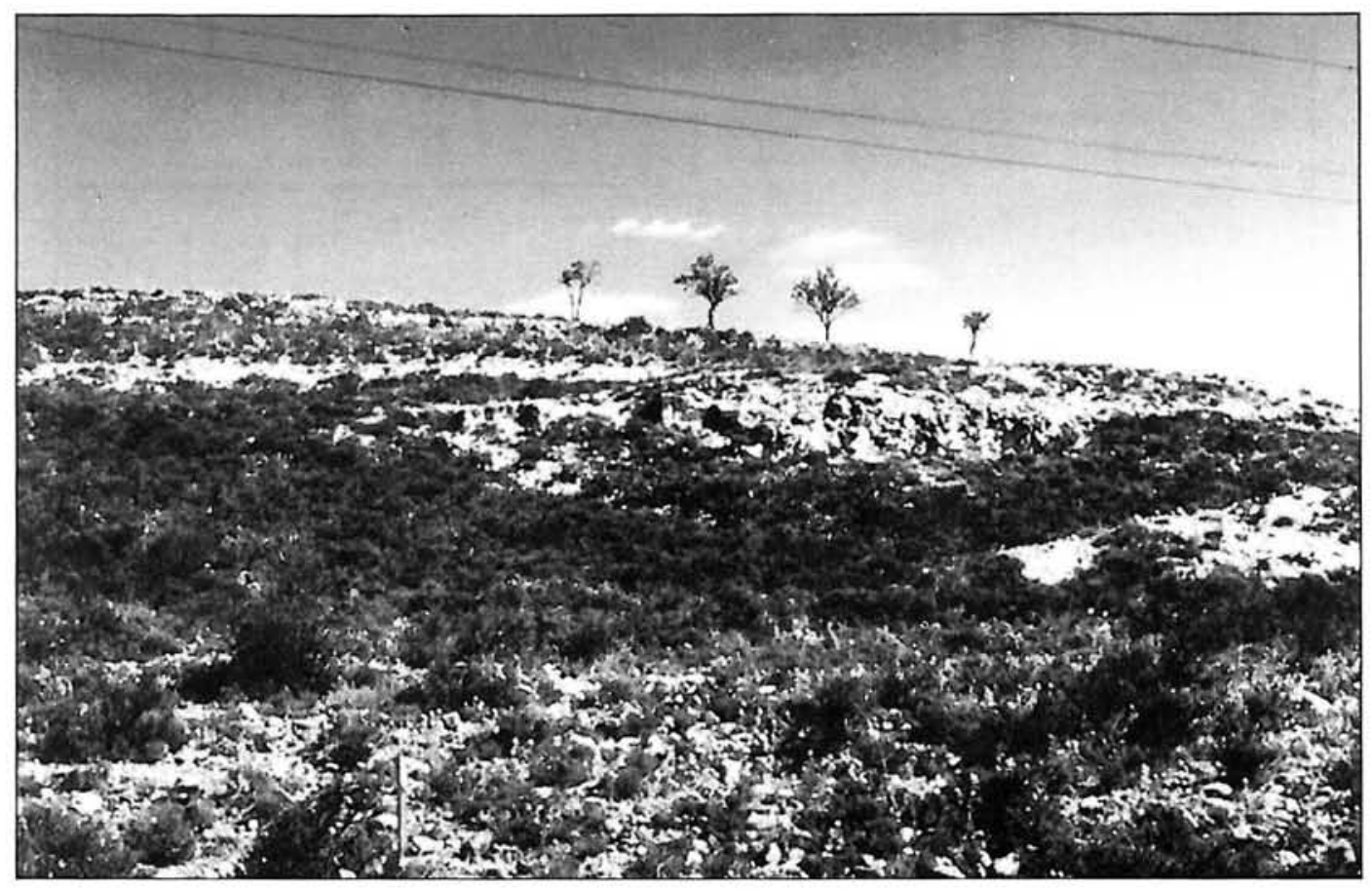

Figura 4. Vista general del frente de la cantera

En el sector Este, sólo se aprecia un pecpueño frente de unos $120 \mathrm{~cm}$, con improntas en el suelo producidas por la separación de dos bloques, uno con longitud de $80 \mathrm{~cm}$ y otro con una de 100 y anchura de 90 ; si bien, estas medidas son aproximadas, debido a la erosión que afecta a toda la cantera.

Ejemplos similares son conocidos en la Península Ibérica, con marcas de extracción, de herramientas, improntas, etc. '.

\section{TRANSPORTE}

La distancia que separa esta cantera del Municipium Augusta Bilbilis apenas es de un kilómetro; ello y otros datos, que posteriormente se aportarán, avalan el uso de este material en el municipio romano.

' Algunos ejemplos y su correspondiente bibliografia son citados en (isneros (unchillos (1988, 59)-80)); para lo casos extranjeros, también muy numerosos puede consultarse la bibliografial yal citada. 


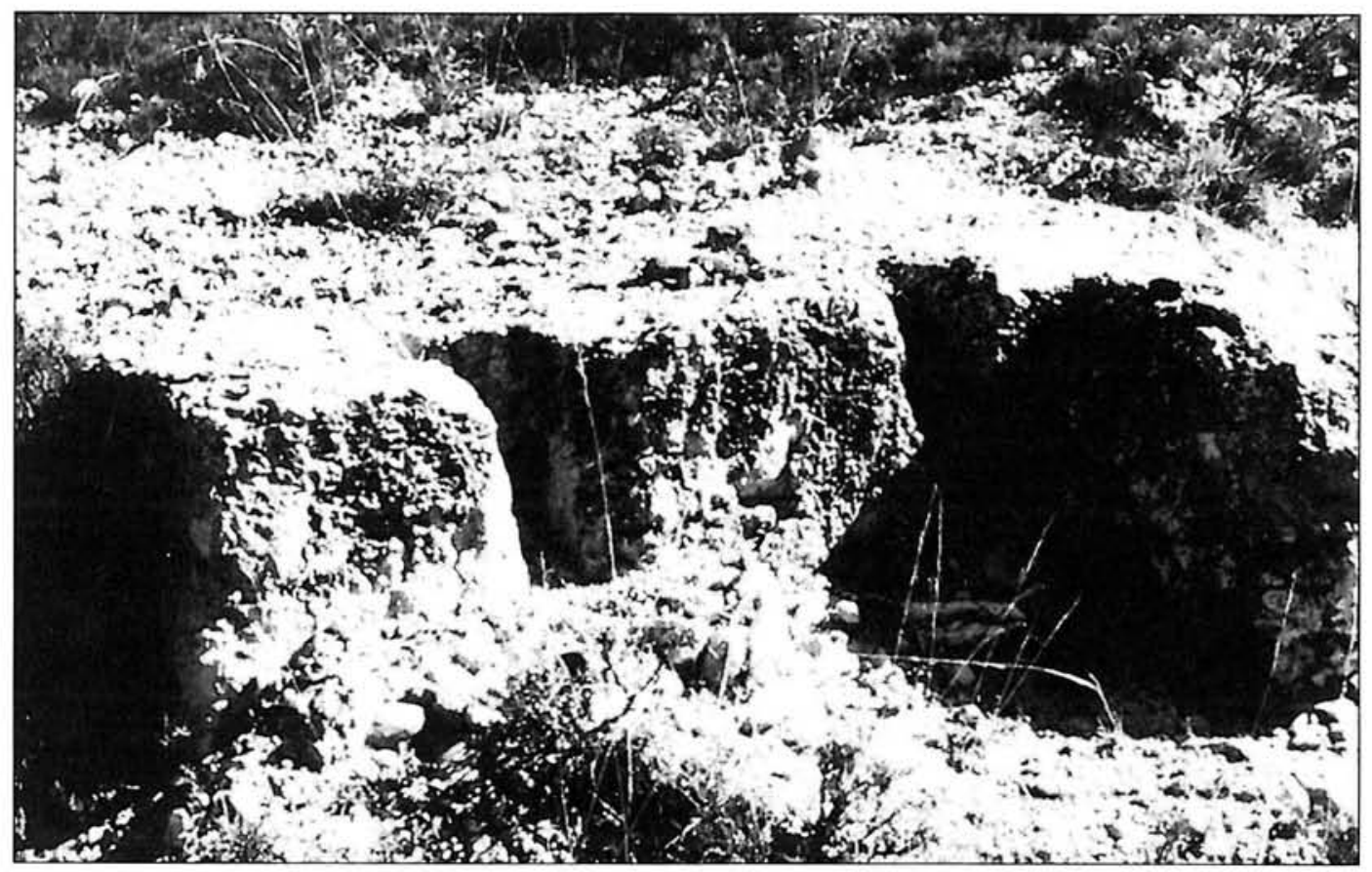

Figura 5. Blecpues en proceso de extraccion

Sin pretender entrar en hipótesis sobre los cliferentes métodos para transportar los bloxues, según su peso y la distancia a recorrer ${ }^{5}$, parece evidente que el acceso de esta materia prima a Bílbilis se efectuó utilizando el entramado viario que rodealsa a la ciudad. A este respecto, se puede recordar su estratégica ubicación, controlando las vías naturales de comunicación del Levante y Norte de Teruel con la Meseta, a través de los ríos Jalón, Jiloca, Perejiles, Ribota y Pieclra, y la existencia de caminos inclígenas utilizados por los romanos (MAGALLÓN BOTAYY, 1987, 189-190).

Otro elemento a valorar es la posilsle existencia de un puente que facilitase el acceso a la ciudad, que en el caso de las calizas de la cantera de Anchís se puede interpretar como obligado, ya que ésta se encuentra en la margen derecha del Jalón, mientras que Bílbilis se halla en la izquierda. Aunque se han efectuado diversas hipótesis sobre dicha ubicación, no hay constancia de ello; si bien J. Liz considera que de haberlo sería en las proximidades de la Torre de Anchís, ya que es aquí donde el río "pasa pegado en su margen derecha a resaltes rocosos que podrían servir de apoyo al puente". Además, basa su hipótesis en la confluencia, en la zona, de varias vías antiguas (Lız. GUIRAI., 1985, 55).

${ }^{3}$ Solbre las diversas formas de transporte, pueden consultarse entre otras obras: Bessac (1980, 143). Mannoni (19812, 103-119), Bedon (1984, 137-139) y Ko\%cli y Wurch Kozelj (1993). 


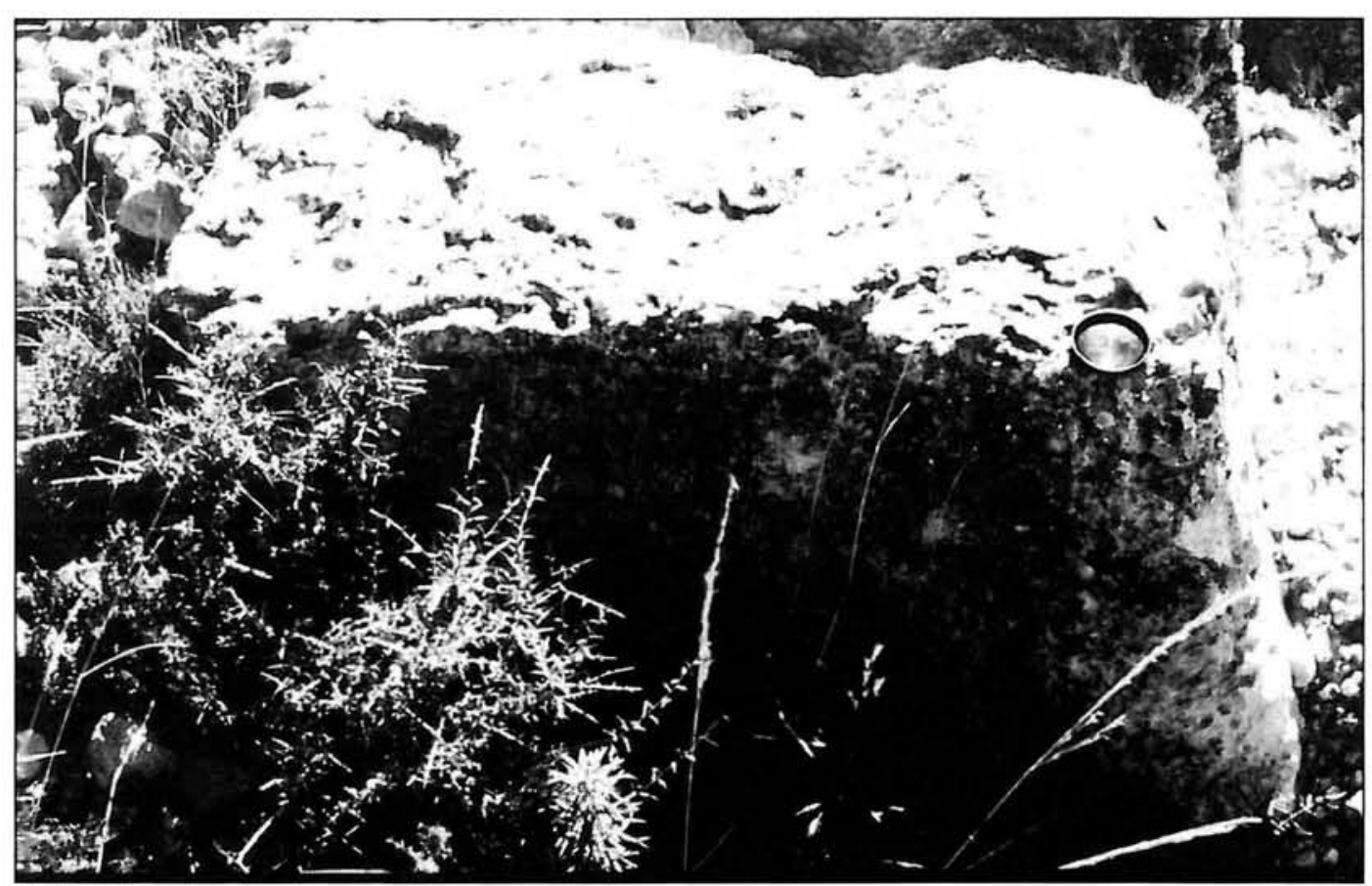

Figura 6. Detalle de uno de los blocues en lase de extracción

\section{TIPOS DE ROCAS Y SU EMPLEO EN BÍLBILIS}

Hay tres grandes tipos de rocas usadas de forma generalizada en los edificios de Bíllbilis:

a) Dolomías, limolitas, areniscas y cuarcitas de las formaciones cámbricas "Dolomías de Ribota" y "Capas del Jalón". Son estas las rocas sobre las que se asienta la ciudad pero estos materiales se utilizan exclusivamente para de mampostería y sillarejo.

b) Sillares de yeso bandeado, que sin lugar a dudas proceden de las rocas terciarias que afloran en los alrededores de Calatayud, empleados con preferencia en esquinas y perfectamente escuadrados.

c) Calizas micríticas con abundante macroporosidad en una facies típica de caliza lacustre localizadas en el teatro —en sillares, capiteles y fustes de columna-, en el foro - en las losas de la plaza-y en las termas — en sillares-. Este tipo de roca es semejante al de la cantera estudiada y en consecuencia realizamos una descripción detallada, a partir de tres muestras tratadas en el laboratorio y algunas decenas de olservaciones de "visu". 


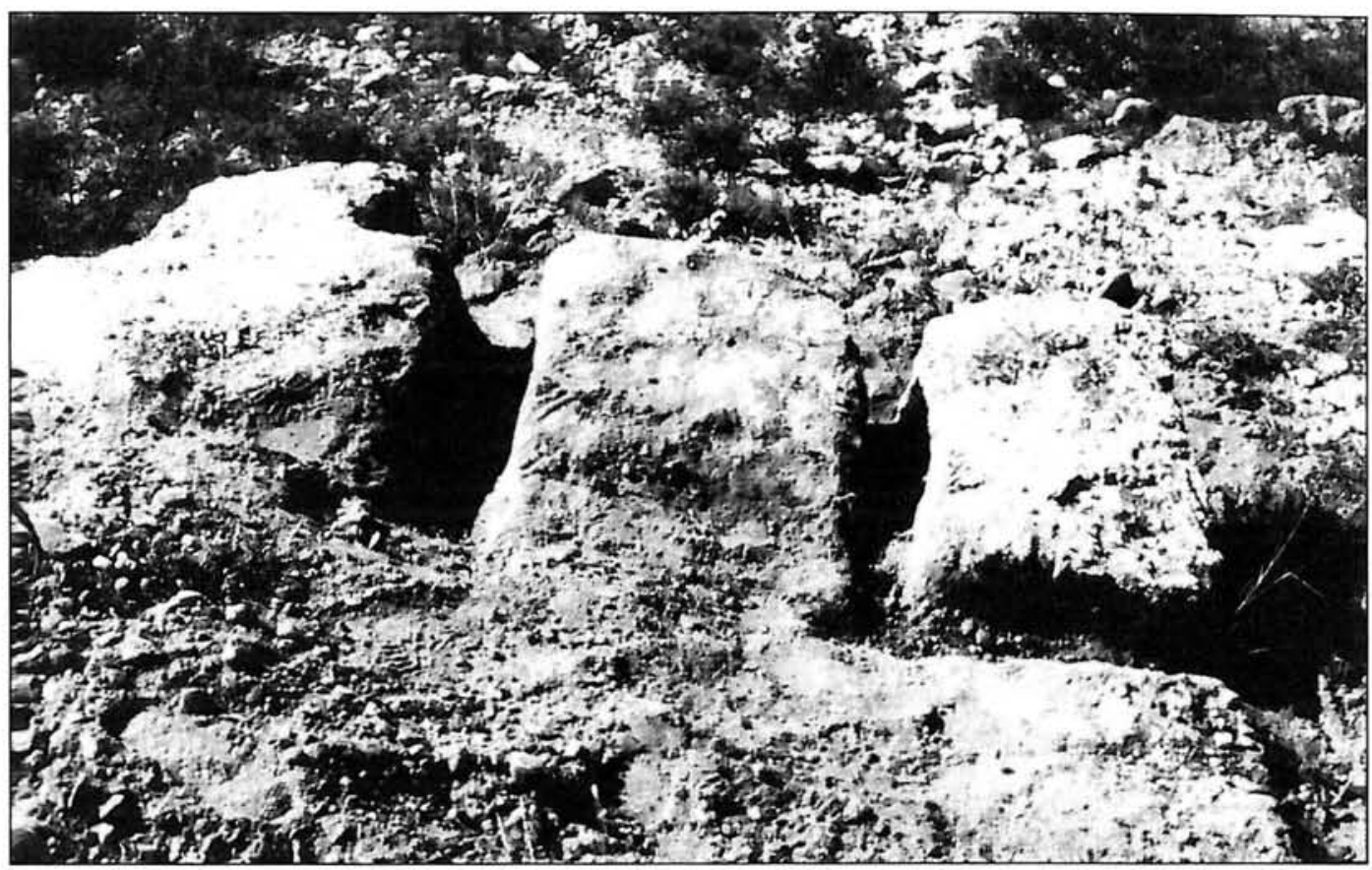

Figura 7. Vista de la cara superior de los blexpes en fase de extración

Facies 1(B-1 sillar muro valva teatro): Caliza travertínica con moldes micríticos de tallos verticales con macroporo central. Entre los moldes de tallos existen intraclastos micríticos (un $20 \%$ con $\mathrm{F}_{\mathrm{m}}=(), 3 \mathrm{~mm}$ ) así como otros macroporos fuera de los moldes vegetales. Hay trazas de terrígenos (cuarzo).

Algunos de los macroporos están rellenos de yeso. Estal característica, más que un carácter primario parece resultado de la presencia de una argamasa de cal y yeso que lleva adherida el material muestreado.Está demostrado que la aplicación de una argamasa con yeso solore una roca (u otro material) conlleva una precipitación de yeso en los poros de la roca a profundiclades variables (DrlicinI) (1996).

Mineralogia: Calcita, yeso, cuarzo

Facies 2(B-2, losa del foro): Caliza micrítica, intramicrita. Intraclastos de $\mathrm{F}_{\mathrm{m}}=0,1$ $\mathrm{mm}$ cle tamaño medio $(20 \%)$ y un $3 \%$ de terrígenos $\left(\mathrm{F}_{\mathrm{m}}=0,08 \mathrm{~mm}\right)$. Afectacla en un $2 \%$ de su superficie por bioturbación por gusanos con tubos de $0,5 \mathrm{~mm}$ de grosor. Macroscópicamente se presenta como muy compacta sin macroporos y con un bandeado gris (discontinuo) paralelo a la estratificación.

Mineralogia:Calcita. En los intraclastos cuarzo, micas y fragmentos de rocas

Facies 3 ( $\mathrm{B}-3$, tambor columna teatro): Caliza arenosa con un $10 \%$ de terrígenos con distribución bimodal de tamaños $\left(\mathrm{F}_{\mathrm{m}}=0,25 \mathrm{~mm}\right.$. y $\mathrm{F}_{\mathrm{m}}=0,05 \mathrm{~mm}$.). Hay grandes intraclastos micríticos que presentan una textura homogénea tan solo perturbacla por hue- 
llas de raíces (o moldes vegetales, en clastos no hay criterios para diferenciar) con macroporos centrales. Los intraclastos son de gran tamaño (bimodales $F_{m}=12 \mathrm{~mm}$ y $F_{m}=4$ $\mathrm{mm})$ y están rodeados por la matriz de micrita y terrígenos. Presenta películas arcillosas alrededor de los intraclastos (gran avidez por el agua, presencia de sepiolita).

Mineralogía: Calcita. En los intraclastos cuarzo, micas y fragmentos de rocas

No cabe duda de que las calizas de Bilbilis son de origen lacustre y las trazas de sepiolita las ubican en el terciario de la cuenca de Calatayud, hecho que por otra parte también encaja con las distancia de afloramiento. En numerosos puntos de las cuencas del Tajo, Duero y Ebro existen rocas semejantes, pero dado que son idénticas a las de la Cuenca de Calatayud es razonable suponer que en época romana se extraían del lugar más próximo. Además de este argumento de la distancia, el composicional de la sepiolita, hace incuestionable esta atribución genérica a esta Cuenca.

En un radio de $5 \mathrm{~km}$ solo existen cuatro zonas (Datos de la cartografía geológica de (TENA, MANDADO y GISBERT, 1980; IGME, 1981) de donde se pudieran extraer calizas. de estas caracteristicas:

1. La Sierra de Armantes.

2.2 Los Altos de la Atalaya (entre los ríos Jalón, Perejiles y el arroyo de la Fuente de la Soga).

3. ${ }^{2}$ La Sierra de Los Arcos delimitada por el río Jalón, la vía férrea Calatayud-Soria y la Rambla de Ribota.

4. ${ }^{\circ}$ Los afloramientos de la cantera estudiada en este trabajo.

En los tres primeros, los afloramientos de calizas ocupan las partes más altas del relieve, siendo ésta su posición estratigráfica habitual ya que son los materiales más modemos dentro de la serie terciaria, mientras que el de la cantera puede considerarse un "lentejón" ya que se ubica en la zona de borde de la Cuenca Terciaria donde habitualmente sólo existen facies detríticas (areniscas y conglomerados), las facies carbonáticas presentes son pues una indentación o cambio de facies dentro del conjunto de facies detríticas de borde.

Los afloramientos 1) y 2) presentan un conjunto de microfacies globalmente distintas de las descritas (calizas algales, calizas dolomíticas y dolomías) (TENA, MANDADO y GISBERT, 1980; IGME 1981) y además están a una distancia (siempre en línea recta) entre 4 y $6 \mathrm{~km}$ de Bilbilis.

Los afloramientos 3) y 4) poseen microfacies semejantes aunque se pueden hacer algunas precisiones sobre las macrofacies.Los estratos calizos de la Sierra de Arcos más próximos al municipio romano, presentan unos grosores $(20-45 \mathrm{~cm})$ inadecuados para la extracción de sillares. Sólo en una situación relativamente Occidental (a unos $3 \mathrm{~km}$ de Bílbilis) existen estratos de más de $60-90 \mathrm{~cm}$. Además, aquellas calizas de la Sierra de Arcos son muy arenosas, (no existiendo rocas de este tipo entre los materiales de construcción empleados en el yacimiento arquelógico). 
Los estratos de la cantera estudiada son, sin embargo, adecuados para la extracción de sillares y piezas de columnas, ya que alcanzan hasta $2 \mathrm{~m}$. de grosor con facies micríticas. Respecto a su semejanza con las muestras estudiadas de Bibilis podemos decir lo siguiente:

Las facies $2 \mathrm{~B}$ es prácticamente idéntica a la facies 1c. La facies $3 \mathrm{~B}$ se asemeja a las facies $2 \mathrm{c}$ y $4 \mathrm{c}$, de hecho posee la textura que cabría esperar en las zonas donde entran en contacto estas dos facies, puesto que es una facies intermedia entre ambas, tal como sucede en la cantera.

La facies 1B no la hemos encontrado en la cantera, aunque en sedimentología de carbonatos lacustres está descrita y asociada verticalmente a la facies $5 c$ que sí está presente en la cantera. No obstante, este tipo de facies aparece en una franja que abarca los afloramientos de Ermita de Ribota-Sierra de Arcos y Altos de La Atalaya. La facies 1B es probable que haya sido extraída en esta banda en la zona más próxima a Bîbilis (Sierra de Arcos).

Por consiguiente existe una notable semejanza entre las microfacies de las rocas empleadas en la ciudad romana y las de la cantera. Si añadimos el hecho de que las tres muestras recogidas en aquélla se tomaron al azar, es decir sin buscar que fueran semejantes a las de la cantera, podemos afirmar que ésta fue, sin duda, fuente de materiales en la construcción de Bibbilis, aunque es evidente que no fue la única.

Por lo que respecta a la metrología, sillares de parecidas dimensiones a los vestigios de la cantera se han hallado en el teatro, con una longitud de entre 72 y $92 \mathrm{~cm}$, un anchura de entre 41 y 48 y una altura de entre 29 y 45 ; de semejantes proporciones son los de las termas. Las losas de la plaza del foro, si bien presentan tamaños diversos, las que en estos momentos se conservan enteras, tienen entre 80 y $86 \mathrm{~cm}$ de longitud y entre 43 y 53 de anchura.

Los sillares, como era habitual, se extraían con dimensiones ligeramente superiones a las de su uso definitivo, para evitar que posibles accidentes en la fase de transporte terrestre los hiciesen inservibles. Por lo que no se deben buscar y comparar medidas exactas entre los restos existentes en la cantera y los sillares de los edificios públicos de Búlbilis, ya que, por otra parte, es arriesgado, en la actualidad, con los bloques en fase de extracción que tenemos intentar dar hipótesis sobre módulos y su aplicación en construcciones.

Además esta roca no estuvo cara vista en los edificios públicos, salvo en el ejemplo de las losas de la plaza pública, por lo que es necesario una limpieza y excavación de la cantera para poder valorar y cuantificar los bloques, improntas y otros restos de extracción y relacionarlos con posterioridad, con los vestigios existentes en el yacimiento.

\section{CONSIDERACIONES FINALES}

Tanto en el teatro -en sillares, capiteles y fustes de columna - como en el foro —en las losas de la plaza - y en las termas —en sillares - fueron empleadas calizas mi- 
críticas con abundante macroporosidad, en una facies típica de caliza lacustre terciaria que sin lugar a dudas procede de la cuenca Neógena de Calatayud.

En la citada cuenca, y a distancias inferiores a 5-6 km de Bílbilis, sólo existen dos afloramientos de rocas semejantes a las empleadas en el municipio romano; la Sierra de Arcos y la cantera estudiada. Ambos lugares pudieron ser fuente de los sillares, aunque los afloramientos adecuados en la Sierra de Arcos están a $2,5 \mathrm{~km}$ del yacimiento y la cantera de Anchía tan sólo a $1,2 \mathrm{~km}$.

Del estudio de microfacies de las rocas se concluye que esta cantera posee una semejanza total con las losas del foro y un gran afinidad con parte de la caliza empleada en capiteles y fustes de columnas del teatro.

La afección a la que se verá sometida por la construcción del AVE es la de su destrucción total, ya que coincide con el trazado de la vía, por lo que como medida correctora esperamos que sea llevada a cabo la propuesta de limpieza y excavación del lugar, para delimitar su extensión y su morfología definitiva, así como una documentación arqueológica completa y detallada. Con ello nuestra pérdida, aunque irreparable, será menor, puesto que la investigación arqueológica española está casi carente de vestigios de este tipo que permiten documentar una fase importante del proceso constructivo de las ciudades hispanorromanas. Asimismo, se podrá paliar, con dicho intervención, una laguna en los conocimientos sobre el municipio bilbilitano.

\section{BIBLIOGRAFIA}

BEDON, R. (1984): Les carrières et les carriers dans le Gaule romaine, París.

BESSAC, J. C. (1980): "Le rempart héllenistique de Saint Blaise (Saint-Mitre-les-Remparts, B. du Rh.): Technique de construction", Documents d'archéologie méridionale 3, 137-157.

CISNEROS CUNCHILlos, M. (1988): Mármoles bispanos: su empleo en la España romana, Zaragoza.

- (1989): "Algunos materiales pétreos utilizados en Bilbilis (Calatayud, Zaragoza)", II Encuentro de Estudios Bilbilitanos, Calatayud (1986), Zaragoza, 61-63.

CisNeros CUNChILlos, M., y MARTiN-BUENo, M. (1994): "El empleo del mármol en el Municipium Augusta Bilbilis): aspectos cuantitativos y decorativos", XIV Congreso Internacional de Arqueologia Clásica, Tarragona (1993), Tarragona, 197-198.

DELGADO, S. (1996): Creación de un banco de materiales (rocas, morteros y ladrillos) con fines de identificación arqueológica y restauración del patrimonio, Informe interno a la Institución Fernando el Católico.Zaragoza.

IGME (1981): Mapa Geológico de España escala 1):50.000. Hoja 409. Calatayud. Madrid. 
Kozeij, T. (1988a): "Extraction of Blocks in Antiquity: special Methods of Analysis", en N.Herz y M.Waelkens (eds.), Classical Marble: Geochemistry, Technology, Trade, Dordrecht-Boston-Londres, 31-39.

- (1988b): "Les carrières des époques grecque, romaine et byzantine", en J.Clayton Fant (ed.), Ancient Marble Quarrying and Trade, BAR International Series 453, Oxford, 3-79.

KozelJ, T., y WURCH Kozel, M. (1993): "Les transports dans l'Antiquitế", en R. Francovich (ed.), Archeologia delle attività stattive e metallurgiche, Florencia, 97-142.

LIz GUIRAL, J. (1985): Puentes romanos en el convento jurídico caesaraugustano, Zaragoza.

MAGALLON BOTAYA, M. ${ }^{2}$ A. (1987); La red viaria romana en Aragón, Zaragoza.

MANNONI, L. y T. (1984²): $l l$ marmo, materia y cultura, Génova.

MARTIN-BUENO, M., y CisNeros CunChiLlos, M. (1985): "Aproximación al estudio de los materiales de construcción romanos de Bílbilis (Calatayud, Zaragoza)", XVII Congreso Nacional de Arqueología, Logrono (1983), Zaragoza, 875-879.

ROCKWELL, P. (1989): Lavorare la pietra, Roma.

TENA J ; MANDADO, J., y GISBERT, J. (1980): Sintesis Geológica de la Cuenca Terciaria de Calatayud Instituto de Estudios Nucleares Madrid .Informe intemo, Madrid. 